\title{
Editorial
}

\section{IAC Standardized Reporting of Breast Fine-Needle Aspiration Biopsy Cytology}

\author{
Andrew S. Field ${ }^{\mathrm{a}}$ Fernando Schmitt ${ }^{\mathrm{b}, \mathrm{c}}$ Philippe Vielh ${ }^{\mathrm{c}}$ \\ a Department of Anatomical Pathology, St. Vincent's Hospital, and Notre Dame University Medical School, \\ Sydney, N.S.W., Australia; ${ }^{b}$ Medical Faculty of Porto University and IPATIMUP, Porto, Portugal; ${ }^{\circ}$ Department of \\ Medicine and Pathology, Laboratoire National de Santé, Dudelange, Luxembourg
}

\section{Key Words}

Standardized reporting - Fine-needle aspiration biopsy .

Core needle biopsy

\begin{abstract}
There have been many changes in the roles of fine-needle aspiration biopsy (FNAB) and core needle biopsy (CNB) in the diagnostic workup of breast lesions in routine breast clinics and in mammographic breast screening programs, as well as changes in the management algorithms utilized and the treatments available, since the $\mathrm{NCl}$ consensus on breast FNAB cytology in 1996. A standardized approach will improve training and performance of FNAB and smear-making techniques, and structured reporting will improve the quality and reproducibility of reports across departments, cities and countries providing a basis for quality assurance measures and improving patient care and facilitating research. Linking cytology reports to management algorithms will increase the clinicians' use of FNAB cytology and where appropriate $C N B$, and enhance the use of ancillary tests for prognostic testing. The IAC recognizes that the local medical infrastructure and resources for breast imaging, biopsy and treatment will influence the diagnostic workup and management of breast disease, but best practice guidelines should be established and modified as required.
\end{abstract}

(c) 2016 S. Karger AG, Basel
The IAC Executive has commenced a process to produce a comprehensive and standardized approach to breast fine-needle aspiration biopsy (FNAB) cytology reporting. The aim is to establish a best practice guideline covering:

1 The indications for breast FNAB cytology

2 The FNAB technique, smear-making and materialhandling procedures

3 A practical, standardized and reproducible reporting system including report requirements, terminology definitions, descriptive terms and categories, structured reports, checklists and formats

4 The appropriate ancillary diagnostic and prognostic tests

5 Correlation with clinical management algorithms

While recognizing that the availability of local medical infrastructure and resources influences the approach to the diagnosis and management of breast disease, it is important to have current best practice guidelines which can then be modified as required. Any recommendations regarding indications for and utility of FNAB and the potential correlation of the diagnostic workup and management of breast lesions should recognize that they will vary with the availability of imaging and treatment infrastructure $[1,2]$. The IAC recommendations will take note of such variations.

\section{KARGER}

(c) 2016 S. Karger AG, Basel

E-Mail karger@karger.com

www.karger.com/acy
Correspondence to: Dr. Andrew S. Field

Department of Anatomical Pathology, St. Vincent's Hospital

390 Victoria Street, Darlinghurst

Sydney, NSW 2010 (Australia)

E-Mail andrew.field@svha.org.au 


\section{Why Do This at This Time?}

It is almost 20 years since the NCI consensus meeting in 1996 [3], and there have been many changes in the diagnostic workup of breast lesions in routine breast clinics and mammographic breast screening programs, and changes in the management algorithms utilized.

FNAB in the developed world is now mainly performed by radiologists rather than cytopathologists, using ultrasound guidance of both palpable and impalpable lesions, which has increased the range of accessible lesions, while accentuating problems in the performance of the FNAB and in making smears. The cytopathologist was immediately aware of any problems in their FNAB technique because they were reporting the slides, while many radiologists do not even have routine contact with the reporting pathologist, who struggles with their poorquality slides [4].

In some centers, liquid-based cytopathology has been introduced to try and overcome the problems with handling of the breast FNAB sample, and this has provided some benefits while introducing greater costs and problems related to the loss of some diagnostic cytological criteria [5].

Rapid onsite evaluation reduces inadequate rates and costs to the system by reducing the need for repeat procedures and triaging cases for ancillary tests, but it requires funding for trained cytologists which has limited its uptake [6].

Increasing use of core needle biopsy (CNB) has virtually replaced FNAB of the breast in some centers and regions, particularly in the mammographic screening program assessment clinics which have an emphasis on the workup of calcifications and whose results have been extrapolated inappropriately into the routine assessment of breast lesions; for example, there is no need to core biopsy a probable cyst or fibrocystic change or fibroadenoma [7]. These two complimentary tests have advantages and disadvantages, and there is a need to establish and recommend their most appropriate use [8].

There have been significant advances in the diagnostic interpretation of breast lesions particularly with tomographic mammography, ultrasound and sonographic mammography, and MRI. There is increasing use of ancillary prognostic testing based on the material derived from breast cancers, and although this has been done mainly on $\mathrm{CNB}$, the availability of molecular testing on small FNAB samples, including cell blocks and material taken directly from smears, races ahead $[9,10]$.

\section{How Has the IAC Breast Group Been Established?}

The Breast Group includes cytopathologists, surgical pathologists, radiologists, surgeons and oncologists (see Appendix). Specific subgroups will look at preanalytical, analytical and postanalytical issues. There will be a literature search in preparation of a bibliography. Draft reviews will be prepared by individuals within these specific groups, leading to the development of recommendations and commentaries, which will be distributed with the drafts throughout the group. Then discussion will occur within the groups utilizing the expertise of members, and a consensus draft will be prepared. This draft will then be published on the IAC internet site, and comments from pathologists and other clinicians will be invited. These comments will be further discussed, and incorporated where the group thinks appropriate into a consensus document.

Drafts of various parts of the structured reporting system were presented at the International congress of $\mathrm{Cy}$ tology in Yokohama in May 2016, and further parts will be presented at the European Congress of Cytology in Liverpool, in October 2016, and the American Society of Cytology meeting in November 2016. These presentations will include areas of debate, and discussion will be invited. There will be discussions then with other bodies representing cytopathologists and cytotechnicians to achieve, if at all possible, an international consensus. Final recommendations will be published by mid-2017, hopefully with the production of an atlas by the end of 2017 based on the material produced by the group.

\section{What Are the Benefits of a Standardized Reporting System?}

A standardized approach with best practice guidelines will improve training in and the performance of FNAB and smear-making techniques, and provide a basis for quality assurance measures within institutions, regions and countries and across international borders. Structured reporting improves the quality, clarity and reproducibility of reports across departments, cities, countries and internationally and will assist patient management and improve breast health care and facilitate research [11]. Linking cytology reporting to management algorithms will enhance the clinicians' use of FNAB cytology and where appropriate core biopsy. Standardized use of cell blocks, immunohistochemistry, in situ hybridization and other molecular tests of prognostic and diagnostic markers will improve patient care. 
Structured reports can be defined as reporting in a format which uses standard headings, definitions, terminology and includes required information. The information required in the report may be a mandatory standard or it may be a recommended guideline $[12,13]$. The aim is that the complete report is reproducible and can be understood internationally by other pathologists and crucially by clinicians and that it guides management. Structured reports are often based on a structured checklist that matches the laboratory and cytopathologist's workflow and is presented in a clear format that conveys information to the clinician. Structured reports use specific terminology for diagnostic categories and do not rely on category numbers, and should include a conclusion containing a specific diagnosis or weighted differential diagnosis (DD) with a discussion of the possible diagnoses. The FNAB cytology report should resemble a breast CNB or any other surgical report.

In FNAB cytology of the breast there will be minimum requirements or a 'minimum data set' within the structured report, which it is suggested will include at least:

- A statement of whether the lesion is completely benign, such as 'no malignant cells are seen'

- A statement of cellularity which in some ways is a measure of the adequacy of the material. This will need discussion regarding the definition of adequacy, and the adequacy of material lacking epithelium, such as cyst contents

- A cytological description including any diagnostic criteria or checklist of features and a brief discussion of the features which support various possible diagnoses

- A conclusion or summary with a standardized descriptive diagnosis of the lesion which should be as specific a diagnosis as possible, or a weighted DD if a specific diagnosis is not possible

- A code or category can be placed in the body of the report but not in the conclusion

Coding or categorization of breast FNAB cytology cases allows a grouping of specific lesions or lesions where specific diagnosis may not be possible. Hopefully it will assist management decisions but it should never be used in isolation of a clear descriptive diagnosis that uses standardized terminology. The risk of using a purely numeric coding system is that it decreases the clinician's understanding of the individual report, but a coding system does assist quality assurance and research.

\section{Categories for Reporting Breast FNAB Cytology}

At an inaugural meeting of the Breast Group members who attended the Yokohama International Congress of Cytology, the use of a 3- or 5-stage coding system was discussed. The consensus was to use a 5 -stage system, which will include:

- Code 1 - Insufficient material

- Code 2 - Benign

- Code 3 - Atypical, probably benign

- Code 4 - Suspicious, probably in situ or invasive carcinoma

- Code 5 - Malignant

Such a system was decided upon by the 1993 Australian National Mammographic Screening Pathology Q Group, but a 5-tier coding system has been used widely internationally. Two of the major areas for debate are the definitions of 'atypia' and 'suspicious for malignancy' $[14,15]$. The IAC reporting system will attempt to define specific criteria or sets of criteria or at least scenarios where atypia is the appropriate diagnosis. These could include:

1 Epithelial hyperplasia with marked dispersal often of columnar cells but minimal nuclear atypia, where the DD is epithelial hyperplasia or low-grade intraduct carcinoma

2 Intraduct papillomas with diagnostic stellate papillary fragments but again marked dispersal, where the DD is low-grade intraduct carcinoma

3 Epithelial hyperplasia with more complex possibly cribriform or micropapillary tissue fragments, where the DD is low-grade intraduct carcinoma

4 Stromal hypercellularity without nuclear atypia or necrosis in otherwise typical fibroadenomas raising the possibility of a low-grade phyllodes tumor

5 Low-cellularity smears with minute epithelial tissue fragments and single cells showing eccentric cytoplasm that raise the DD of lobular carcinoma or lobular carcinoma in situ

\section{Establishing an Approach to a Standardized Report for Breast FNAB Cytology}

The IAC Breast Group will establish a checklist for FNAB cytology of the breast using an analytical approach based on pattern recognition and cytological diagnostic criteria that can be used by the reporting cytopathologist and that will generate the report [14-16]. 


\section{Correlation with Management Guidelines}

The FNAB cytology report should be used in conjunction with the clinical and imaging findings in the 'triple test' approach, which yields very high positive and negative predictive values, and provides a solid basis for management decisions in breast lesions. The Breast Group will seek to establish suggested best practice protocols for the workup of each of the 5 structured reporting categories, while taking into account the vast differences between the developed and developing world in the potential imaging and core biopsy infrastructure available $[1,2$, $16,17]$.

Finally, the Breast Group hopes the cytopathology community will assist in the development of breast FNAB cytology guidelines by joining in the debate at the various presentations in the near future, and by critiquing the draft documents when they are placed on the IAC site later this year.

\section{Appendix}

The IAC Breast Group includes Wendy Raymond (Australia), Colleen Wright (South Africa), Shahin Sayed (Kenya), Amy Li (USA), Torill Sauer (Norway), Benjaporn Chaiwun (Thailand), Bob Osamura (Japan), Len Chan (China), Fernando Schmitt (Portugal and Luxembourg), Philippe Vielh (France and Luxembourg), Mary Rickard (Australia), Gary Tse (Hong Kong), Puay Hoon (Singapore), Britt-Marie Ljung (USA), Fraser Symmans (USA), Andrew Lee (UK), Luigi Di Bonito (Italy), Ben Anderson (USA), Giuseppe Curigliano (Italy), Angela Chong (Singapore) and Andrew S. Field (Australia).

\section{Disclosure Statement}

The authors have no conflicts of interest.

\section{References}

1 Masood S, Vass L, Ibarra JA Jr: Breast pathology guideline implementation in low- and middle-income countries. Cancer 2008;113: 2297-2304.

2 Anderson BO: FNAB for breast cancer diagnosis: one size does not fit all. J Natl Compr Canc Netw 2016;14:599-600.

3 Abati A, Abele J, et al: The uniform approach to breast fine needle aspiration biopsy. Diagn Cytopathol 1997;16:295-311.

4 Ljung BM, Drejet A, Chiampi N, et al: Diagnostic accuracy of FNAB is determined by physician training in sampling technique. Cancer (Cancer Cytopathol) 2001;93:263268.

5 Gerhard R, Schmitt FC: Liquid-based cytology in FNAB of breast lesions. Acta Cytol 2014; 58:533-542.

6 Lieu D: Value of cytopathologist performed US guided FNAB as a screening test for US guided core needle biopsy in nonpalpable breast lesions. Diagn Cytopathol 2009;37: 262-269.
7 Nassar A: Core needle biopsy versus FNAB in breast: a historical perspective and opportunities in the modern era. Diagn Cytopathol 2011;39:380-388

8 Ly A, Ono JC, Hughes KS, Pitman MB, Balassanian R: FNAB of palpable breast masses: patterns of clinical use and patient experience. J Natl Compr Canc Netw 2016;14:527-536.

9 Symmans WF, Ayers M, Clark EA, et al: Total RNA yield and microarray gene expression profiles from fine needle aspiration biopsy and core needle biopsy samples of breast carcinoma. Cancer 2003;97:2960-2971.

10 Schmitt F, Vielh P: FNAC samples: a good source of material to evaluate biomarkers in breast cancer. Histopathology 2014;64:971980.

11 Ellis DW, Srigley J: Does standardised structured reporting contribute to quality in diagnostic pathology? The importance of evidence-based data sets. Virchows Arch 2016; 468:51-59.
12 Royal College of Australasia (RCPA): Structured pathology reporting of cancer. https:// www.rcpa.edu.au/Health-Care (accessed October 31, 2016)

13 International Confederation Cancer Reporting (ICCR). https://www.iccr-cancer.org (accessed October 31, 2016).

14 Orell SR, Sterrett GF: Breast; in Orell SR, Sterrett GF (eds): Fine Needle Aspiration Cytology, ed 5. Toronto, Elsevier, 2012, chapt 7.

15 Field AS, Zarka MA: Breast; in Field AS, Zarka MA (eds): Practical Cytopathology: A Diagnostic Approach to Fine Needle Aspiration Biopsy. Amsterdam, Elsevier, 2017, chapt 5.

16 Tse G, Tan PH, Schmitt F. Fine Needle Aspiration Cytology of the Breast. Berlin, Springer, 2013.

17 Ukah Co, Oluwasola OA: The clinical effectiveness of FNAB in patients with palpable breast lesions seen at the University College Hospital, Ibadan, Nigeria: a ten year retrospective study. J Cytol 2011;28:111-113. 